\title{
Ginger Supplementation in Nonalcoholic Fatty Liver Disease: A Randomized, Double-Blind, Placebo-Controlled Pilot Study
}

\author{
Mehran Rahimlou, ${ }^{1,2}$ Zahra Yari, ${ }^{3}$ Azita Hekmatdoost, ${ }^{4}$ Seyed Moayed Alavian, ${ }^{2}$ and Seyed \\ Ali Keshavarz," \\ ${ }^{1}$ Department of Community Nutrition, School of Nutritional Sciences and Dietetics, Tehran University of Medical Sciences (TUMS), Tehran, IR Iran \\ ${ }^{2}$ Baqiyatallah Research Center for Gastroenterology and Liver Diseases, Baqiyatallh University of Medical Sciences, Tehran, IR Iran \\ 3 Department of Nutrition, Faculty of Nutrition and Dietetics, Shahid Beheshti University of Medical Sciences, Tehran, IR Iran \\ 4 Department of Clinical Nutrition and Dietetics, Faculty of Nutrition and Food Technology, National Nutrition and Food Technology Research Institute, Shahid Beheshti University \\ of Medical Sciences, Tehran, IR Iran \\ ${ }^{5}$ Department of Clinical Nutrition, School of Nutritional Sciences and Dietetics, Tehran University of Medical Sciences (TUMS), Tehran, IR Iran \\ ${ }^{*}$ Corresponding Author: Seyed Ali Keshavarz, Department of Clinical Nutrition, School of Nutritional Sciences and Dietetics, Tehran University of Medical Sciences (TUMS), Tehran, IR \\ Iran. Tel: +98-88973901; +98-88973905, E-mail: s_akeshavarz@yahoo.com
}

Received 2015 November 20; Revised 2015 December 6; Accepted 2015 December 7.

\begin{abstract}
Background: Nonalcoholic fatty liver disease (NAFLD) is one of the most common chronic liver diseases worldwide. The pathogenesis of this disease is closely associated with obesity and insulin resistance. Ginger can have hypolipidemic and antioxidant effects, and act as an insulinsensitizer.

Objectives: The aim of this study was to evaluate the effects of ginger supplementation in NAFLD management.

Patients and Methods: In a randomized, double-blind, placebo-controlled clinical trial, 44 patients with NAFLD were assigned to take either two grams per day of a ginger supplement or the identical placebo, for 12 weeks. In both groups, patients were advised to follow a modified diet and physical activity program. The metabolic parameters and indicators of liver damage were measured at study baseline and after the 12 week intervention.

Results: Ginger supplementation resulted in a significant reduction in alanine aminotransferase, $\gamma$-glutamyl transferase, inflammatory cytokines, as well as the insulin resistance index and hepatic steatosis grade in comparison to the placebo. We did not find any significant effect of taking ginger supplements on hepatic fibrosis and aspartate aminotransferase.

Conclusions: Twelve weeks of two grams of ginger supplementation showed beneficial effects on some NAFLD characteristics. Further studies are recommended to assess the long-term supplementation effects.
\end{abstract}

Keywords: Nonalcoholic Fatty Liver Disease, Ginger, Hepatic Steatosis, Inflammatory Biomarkers, Lipid Profile

\section{Background}

Nonalcoholic fatty liver disease (NAFLD) is one of the most common chronic liver diseases worldwide and occurs predominantly in obese, sedentary people and patients with type II diabetes (1). NAFLD includes a wide variety of liver disorders from simple steatosis to hepatic fibrosis and cirrhosis (2). It is usually associated with metabolic disorders such as obesity, insulin resistance (3), hypertension, dyslipidemia and impaired fat metabolism (4), and it can increase mortality risk due to the associated cardiovascular disease (5). Although there is no proven treatment for this disorder, researchers have suggested a combination of modified diet and exercise for treatment (6). Previous studies have shown that a diet rich in antioxidants and anti-inflammatory agents can be effective in the treatment of NAFLD (7).

Ginger is the root of Zingiber officinale and is one of the most used spices in many countries. Ginger contains ac- tive ingredients such as gingerol, shogaol, zingerone and $\beta$-bisabolene (8). In ancient medical practice, ginger was used for treatment of various disorders such as rheumatoid arthritis, neurodegenerative diseases, inflammation and asthma (9). Previous studies have shown that ginger and its active compounds can exhibit anti-diabetes, anticancer and anti-inflammatory properties $(10,11)$. It has been shown that ginger extract can exhibit antioxidant activity and reduce the levels of pro-inflammatory biomarkers (12). Moreover, recent studies on patients with Type II diabetes and hyperlipidemia have shown that ginger can reduce insulin resistance and serum triglyceride concentration $(13,14)$.

\section{Objectives}

Accordingly, the hypothesis of this study was that ginger

Copyright (C) 2016, Kowsar Corp. This is an open-access article distributed under the terms of the Creative Commons Attribution-NonCommercial 4.0 International License (http://creativecommons.org/licenses/by-nc/4.0/) which permits copy and redistribute the material just in noncommercial usages, provided the original work is properly cited. 
supplementation can be introduced as a new therapeutic strategy for NAFLD by improving the body's antioxidant activity, and reducing levels of inflammation and insulin resistance. A randomized double-blind study was designed to evaluate this hypothesis in order to examine the effectiveness of ginger supplementation in patients with NAFLD.

\section{Patients and Methods}

\subsection{Participants}

Patients were identified and recruited from the Baqiatallah hospital, Iran. Inclusion criteria included the presence of steatosis on fibroscan (CAP score > 270) and a high concentration of liver enzyme (alanine aminotransferase (ALT) greater than 1.5 times the upper limit of the normal range) at the time of randomization. Exclusion criteria included chronic liver diseases such as various types of hepatitis, diabetes mellitus, cancer, inherited disorders affecting the liver condition (storage disorders of iron, copper, and others), non-treated hypothyroidism, as well as, alcohol use, taking any medications, weight loss surgery in the last year or strict diets to lose weight in the past three months, pregnancy, lactation, autoimmune diseases, weight loss more than ten percent of initial body weight during the intervention, unwillingness to continue the study protocol, and not taking more than ten percent of supplements in any follow-up visit.

\subsection{Study Design}

Eligible patients with NAFLD were invited to participate in this study. At first, all participants were informed about the study protocol by being given a complete description of the objectives, benefits and potential harms of the study. The study was approved by the ethics committee of Tehran University of Medical Sciences and informed consent was received from the participants who elected to participate in the study. Eventually 50 patients who met the inclusion criteria were selected.

Participants were randomly allocated based on age, sex and severity of steatosis into two groups. Randomization lists were computer-generated by a statistician and participants, project managers and employees at the clinic were completely unaware (blind) about the intervention and control groups. At the first visit, baseline data were gathered and patients were advised to consume two capsules, each containing $500 \mathrm{mg}$ of ginger (Green Plants of Life Pharmaceutics Co., Iran) or identical placebos (starch) one hour after breakfast and two capsules after dinner for 12 weeks. For all patients, capsules were given in the first week for three weeks, and in each visit a new series of supplements were prescribed. In both groups, patients were advised to follow a modified diet and physical activity plan, according to the clinical guidelines on the Identification, evaluation, and treatment of overweight and obesity in adults from the national institutes for health $(\mathrm{NIH})$ and the north American association for the study of obesity (15). Ac- cording to these groups, less than $30 \%$ of energy from lipids (10\% from SFAs, $15 \%$ from MUFAs and 5\% from PUFAs), $15 \%$ to $18 \%$ of energy from proteins and 52\% to 55\% of energy from carbohydrates were provided. Also, patients were advised to limit their dietary cholesterol intake to less than $300 \mathrm{mg}$ per day and consume 20 to 30 grams of fiber per day. All patients were advised to exercise $\geq 30 \mathrm{~min}$, three times per week. To remind the participants about supplement consumption phone calls were made at the end of each week.

\subsection{Clinical, Paraclinical, and Dietary Intake Assessments}

For all patients, anthropometric parameters, including weight, height, waist and hip circumference, were measured at the beginning (week 0) and the end (week 12) of the study. body mass index (BMI) was calculated by dividing weight to height squared $\left(\mathrm{m}^{2}\right)$, and waist to hip ratio (WHR) was calculated by dividing the waist to hip circumference. All the measurements were made by one person in order to reduce measurement errors. To assess food intake, all participants of the study were asked to complete a three-day food record at the beginning and at the end of the study. Blood samples were received from all patients after 10 to 12 hours of fasting at the beginning (week 0) and the end (week 12) of the study.

All biochemical tests were accurately measured in the same laboratory. G-glutamyltransferase (GGT) was measured using an enzymatic colorimetric assay (Parsazmoun). ALT and aspartate aminotransferase (AST) concentrations were measured using a photometric assay (Parsazmoun). The concentration of TNF- $\alpha$ was measured using an ELISA kit (eBioscience, Inc., San Diego, CA, USA, Cat NO: 109957025) and high-sensitivity C-reactive protein concentration was also measured by ELISA kit (Parsazmun Co., Tehran, Iran, Cat NO: 93001). Fasting blood glucose levels were measured using the GOD/POD method. Serum insulin concentrations were measured using an ELISA kit (Monobind, Inc., Lake FOREST, CA, USA, Catalog NO:58K1L4). Insulin resistance was measured by the homeostasis model formula (HOMA-IR-IR) as follows: HOMA-IR = [fasting insulin $(\mathrm{mU} / \mathrm{L}) \times 3$ fasting blood glucose $(\mathrm{mg} / \mathrm{dL})] / 405$ (16). The steatosis and hepatic fibrosis in patients was measured using ultrasound elastography at the beginning and the end of the study. The physical activity of patients was measured by the metabolic equivalent of task (MET) questionnaire at the beginning and the end of the study.

\subsection{Statistical Analysis}

Statistical analysis was performed using SPSS version 19. Normal distribution of data was assured using the Kolmogorov-Smirnov and Shapiro-Wilk tests. Normally distributed variables are reported as mean \pm standard deviation. Baseline characteristics of variables were compared among the two groups using independent sample t-test, Mann-Whitney U test and chi-square test. Withingroup comparisons, before and after the intervention, 
were made with the Student's t-test or Wilcoxon. In order to eliminate the effects of confounding factors, the analysis of covariance test was used. All ANCOVA models were adjusted for the baseline value of each variable and mean changes in BMI, WHR, MET and energy.

This trial was registered at clinicaltrials.gov as NCT02535195

\section{Results}

\subsection{Participants' Characteristics}

Fifty patients were included in the study and were randomly assigned to take either two grams of ginger $(\mathrm{n}=$ 25 ) or the identical placebo $(n=25)$. Two patients in the ginger group (due to unwillingness to continue) and four patients in the control group (two persons due to immigration and two persons due to unwillingness to continue) were excluded, and 44 people, including 23 patients in the ginger group and 21 patients in the control group were entered into the final analysis. Demographic and clinical characteristics are summarized in Table 1. Eighty-eight percent of patients in the study successfully completed 12 weeks of treatment. No significant difference was found between demographic index levels, weight, BMI, nutritional intake (energy, selenium, vitamin D, vitamin E, vitamin C, omega-3 and omega-6), physical activity, and especially, the steatosis degree and liver fibrosis between the two groups at baseline. However, there was a significant difference between the two groups in levels of hs-CRP and AST liver enzyme at the beginning of the study.

\subsection{Primary Outcome}

Serum levels of the ALT in both groups had a significant reduction $(\mathrm{P}<0.05)$, but it declined significantly more in the ginger group compared with the placebo group $(\mathrm{P}=$ 0.02 ) (Table 2). Also, it remained significant after adjusting the results for the energy, physical activity, WHR and BMI variables $(\mathrm{P}=0.045)$ (Table 3$)$.

Table 1. Baseline Characteristics at Enrollmenta ${ }^{a}$

\begin{tabular}{|c|c|c|c|c|}
\hline Variables & Total $(n=44)$ & Ginger Group $(n=23)$ & Placebo Group $(n=21)$ & P Value \\
\hline Age, $y$ & $45.22 \pm 1.55$ & $45.45 \pm 2.31$ & $45 \pm 2.14$ & 0.886 \\
\hline Gender & & & & 0.405 \\
\hline Male & 20 & 11 & 9 & \\
\hline Female & 24 & 11 & 13 & \\
\hline Smoking & & & & 1.000 \\
\hline Yes & 4 & 2 & 2 & \\
\hline No & 41 & 20 & 20 & \\
\hline \multicolumn{5}{|l|}{ Metabolic characteristics } \\
\hline Weight, kg & $83.92 \pm 2.33$ & $86.47 \pm 3.59$ & $81.38 \pm 2.96$ & 0.285 \\
\hline Height, cm & $164.21 \pm 1.71$ & $168.33 \pm 2.06$ & $160.14 \pm 2.48$ & 0.015 \\
\hline BMI, $\mathrm{kg} / \mathrm{cm}^{2}$ & $31.04 \pm 0.54$ & $30.55 \pm 0.95$ & $31.53 \pm 0.47$ & 0.280 \\
\hline Waist circumference, cm & $103.47 \pm 1.26$ & $104.4 \pm 2.19$ & $102.54 \pm 1.29$ & 0.323 \\
\hline Hip circumference, $\mathrm{cm}$ & $108.15 \pm 0.92$ & $109.59 \pm 1.46$ & $106.72 \pm 1.07$ & 0.122 \\
\hline WHR & $0.95 \pm 0.009$ & $0.95 \pm 0.01$ & $0.96 \pm 0.01$ & 0.543 \\
\hline MET, h/d & $31.47 \pm 0.6$ & $32.02 \pm 4.1$ & $32.02 \pm 4.2$ & 0.598 \\
\hline Energy, kcal & $2329.92 \pm 61.15$ & $2447.52 \pm 82.88$ & $2212.31 \pm 84.42$ & 0.053 \\
\hline
\end{tabular}

Abbreviations: BMI, body mass index; MET, metabolic equivalent of task; WHR, waist to hip ratio.

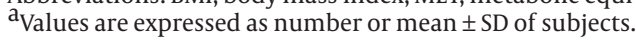

Table 2. Means and Standard Deviations of the Outcome Measures ${ }^{\mathrm{a}}$

\begin{tabular}{|c|c|c|c|c|c|c|c|}
\hline \multirow[t]{2}{*}{ Variables } & \multicolumn{2}{|c|}{ Intervention Group } & \multicolumn{2}{|c|}{ Placebo Group } & \multicolumn{3}{|c|}{ PValue $^{b, c}$} \\
\hline & Before & After & Before & After & $\mathbf{P}_{1}$ & $\mathbf{P}_{2}$ & $\mathbf{P}_{\mathbf{t}}$ \\
\hline ALT, $\mathbf{U} / \mathbf{L}$ & $36.59 \pm 0.58$ & $30.5 \pm 0.97$ & $34.53 \pm 0.29$ & $30.82 \pm 0.74$ & .002 & 0.002 & .02 \\
\hline AST, $\mathbf{U} / \mathbf{L}$ & $36.04 \pm 0.69$ & $31.67 \pm 0.79$ & $33.44 \pm 0.49$ & $29.48 \pm 0.91$ & .005 & .003 & .02 \\
\hline GGT, U/L & $40.32 \pm 2.06$ & $30.26 \pm 1.64$ & $44.45 \pm 0.26$ & $41.82 \pm 0.35$ & .026 & .022 & .01 \\
\hline HOMA-IR & $2.93 \pm 0.34$ & $2.19 \pm 0.3$ & $2.69 \pm 0.22$ & $2.38 \pm 0.2$ & .002 & .005 & .01 \\
\hline Fibrosis score, KPa & $7.02 \pm 0.43$ & $5.09 \pm 0.41$ & $7.92 \pm 0.46$ & $7.15 \pm 0.45$ & .001 & .009 & .335 \\
\hline Steatosis score, CAP & $307.81 \pm 8.007$ & $255.77 \pm 7.86$ & $287.45 \pm 7.17$ & $276.36 \pm 7.77$ & .004 & .007 & .022 \\
\hline hs-CRP, ng/mL & $4.62 \pm 0.14$ & $3.42 \pm 0.12$ & $4.79 \pm 0.18$ & $4.44 \pm 0.16$ & .007 & .005 & .032 \\
\hline TNF- $\alpha, p g / m L$ & $4.68 \pm 0.37$ & $3.52 \pm 0.38$ & $3.03 \pm 0.21$ & $2.88 \pm 0.21$ & 0 & .003 & .002 \\
\hline
\end{tabular}

Abbreviations: ALT, alanine aminotransferase; AST, aspartate aminotransferase; GGT, $\gamma$-glutamyl transferase; HOMA-IR, homeostasis model formula for insulin resistance; hs-CRP, high-sensitivity C-reactive protein; TNF- $\alpha$, tumor necrosis factor alpha.

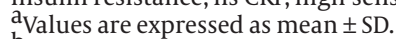

$\mathrm{b}_{\mathrm{P} 1}$ and P2 value, difference compared with the value at the beginning of the study within groups (paired t-test).

${ }^{\mathrm{C}} \mathrm{Pt}$ value, mean difference of changes between the two groups (independent $\mathrm{t}$-test). 
Rahimlou M et al.

Table 3. Adjusted Mean Changes and Standard Deviation of the Outcome

\begin{tabular}{lccc}
\hline Variable & Ginger Group & Placebo Group & PValue \\
\hline ALT & $-5.81 \pm 4.05$ & $-3.71 \pm 2.97$ & 0.045 \\
AST & $-4.36 \pm 2.93$ & $-3.95 \pm 4.07$ & 0.866 \\
GGT & $-10.05 \pm 9.25$ & $-2.62 \pm 1.77$ & 0.001 \\
HOMA-IR & $-0.73 \pm 0.85$ & $-0.31 \pm 0.24$ & 0.022 \\
hs-CRP & $-0.36 \pm 0.24$ & $-0.17 \pm 0.09$ & 0.016 \\
TNF- $\alpha$ & $-1.15 \pm 0.67$ & $-0.14 \pm 0.05$ & 0.01 \\
Steatosis score & $-52.04 \pm 36.33$ & $-11.09 \pm 5.69$ & $<0.001$ \\
Fibrosis score & $-1.12 \pm 1.5$ & $-0.77 \pm 1.2$ & 0.39 \\
\hline
\end{tabular}

Abbreviations: ALT, alanine aminotransferase; AST, aspartate aminotransferase; GGT, $\gamma$-glutamyl transferase; HOMA-IR, homeostasis model formula for insulin resistance; hs-CRP, high-sensitivity C-reactive protein; TNF- $\alpha$, tumor necrosis factor alpha.

\subsection{Secondary Outcomes}

Anthropometric measures, including weight, BMI, waist circumference, hip circumference and waist-to-hip ratio, decreased significantly in both groups at the end of study, but this reduction was not significantly different between the two groups except for hip circumference, which reduced significantly more in the ginger group in comparison to the placebo group $(\mathrm{P}=0.015)$. Improvements in serum levels of AST and GGT were observed in both groups; however, this reduction was significant only in the ginger group. After adjusting the results for baseline values, this reduction was significant only for GGT $(\mathrm{P}<0.01)$, and AST changes between the two groups was not significant $(\mathrm{P}=0.886)$. A substantial improvement in most liver indicators was observed within each group and between the two groups before and after the adjustment for confounder variables. Patients taking ginger capsules compared to the placebo group had a significantly greater decrease in the steatosis score $(\mathrm{P}<$ 0.001), but the difference in fibrosis score between the two groups was not significant $(\mathrm{P}=0.39)$. At the end of the study, there was a significant reduction in levels of TNF- $\alpha$ and hs-CRP in both groups, but the decline was greater in the ginger group ( 0.01 and 0.016 , respectively). Furthermore, the ginger supplement compared to the placebo caused greater reduction in the levels of insulin resistance index (HOMA-IR $)(\mathrm{P}=0.022)$.

No severe effects were reported due to the ginger or placebo supplementation by the patients. Only one patient complained of mild headache and one of heartburn caused by the ginger supplements, both of which were resolved by recommendations of the nutritional consultants.

\section{Discussion}

To our knowledge, this is the first randomized, doubleblind clinical trial study that examined the effects of ginger supplementation with lifestyle intervention on liver enzymes, inflammatory markers, steatosis and hepatic fibrosis scores in patients with nonalcoholic fatty liver disease
(NAFLD). In this study, daily consumption of two grams of ginger supplement for 12 weeks resulted in a significant decrease in inflammatory marker levels, ALT and GGT liver enzymes and liver steatosis. Insulin resistance is one of the major risk factors in the pathogenesis of NAFLD (16). Our study has shown that ginger supplementation reduces insulin resistance, which is in line with previous studies.

Shidfar et al. have shown that ginger supplementation can ameliorate levels of insulin resistance markers (14). Furthermore, Mozaffari et al. showed that a daily intake of three grams of ginger powder for eight weeks resulted in a significant reduction in fasting blood sugar levels and insulin resistance (17). Thus, our study confirmed these previous studies, and showed that as little as two grams/ day of ginger can reduce insulin resistance. According to the other studies, inflammatory agents play an important role in the pathogenesis of NAFLD. Some studies have shown that in patients with NAFLD, polymorphisms of TNF- $\alpha$ promoter, at positions 238 (TNFA) and 308 (TNF2), are common and its expression increases (18). TNF-a plays a critical role in both insulin resistance and liver fibrosis and increases fatty acids oxidation $(19,20)$. So, reduction of TNF- $\alpha$ and other inflammatory factors can improve the treatment of NAFLD. In this study, it was shown that ginger supplementation caused a significant decrease in serum levels of TNF- $\alpha$ and hs-CRP, which is consistent with previous studies such as those by Lee et al. (21) and Habib et al. (22). After the adjustment for confounders, the hepatic steatosis score in the group receiving ginger supplements reduced significantly more than the control group, while liver fibrosis showed no significant difference between the two groups. Hepatic steatosis is characterized by overload of triglycerides in hepatocytes that can injure the liver (23). Animal studies have shown that the active compounds in ginger can enhance the antioxidant defense systems, such as glutathione peroxidase and glutathione S-transferase, and reduce levels of malondialdehyde (MDA) and hepatic steatosis $(24,25)$. It seems that one of the main reasons for this protective feature is the effect of ginger on the expression of proliferating cell nuclear antigen (PCNA). PCNA is a nuclear protein that is involved in regulating cell proliferation. In liver disorders such as NAFLD and cirrhosis, the expression of PCNA is increased, which ultimately can result in excessive and uncontrolled proliferation of tissue to replace the damaged tissue and causes liver fibrosis progression. Previous studies have shown that ginger supplementation can reduce the expression of PCNA and prevent the intensification of liver fibrosis (26). In this study, no significant decrease was observed in liver fibrosis score in the ginger group compared to the placebo group. This can be due to the short-term of the intervention, because it takes a long time for regeneration of hepatic tissue and reduction of fibrotic tissue.

The lack of using liver biopsy to derive a pathology score of disease was one of the limitations of this study. Researchers were not able to use this method in this study due to its invasive nature, however, we evaluated 
Rahimlou M et al.

the hepatic steatosis and fibrosis with transient elastography, which provides quantitative, noninvasive results. Another limitation of this study was its short intervention time. The advantage of this study was that it is the first study evaluating the effects of ginger supplementation on NAFLD.

In conclusion, this randomized controlled clinical trial found some evidence that ginger supplementation could increase the effectiveness of lifestyle interventions (diet modification and physical activity) compared with lifestyle interventions alone for treatment of NAFLD. Whether these effects will be sustained and/or augmented with longer treatment durations remains to be determined.

\section{Acknowledgments}

We thank green plants of life pharmaceutics Co. for providing ginger and placebo capsules and all participants that contributed to this study.

\section{Footnotes}

Authors' Contribution:Study concept and design: Seyed Ali Keshavarz; acquisition of data: Mehran Rahimlou; analysis and interpretation of data: Seyed Moayed Alavian; drafting of the manuscript: Azita Hekmatdoost; critical revision of the manuscript for important intellectual content: Zahra Yari.

Funding/Support:This work was supported by Tehran University of Medical Sciences (grant ID: 93-03-161-27265).

\section{References}

1. Ong JP, Younossi ZM. Epidemiology and natural history of NAFLD and NASH. Clin Liver Dis. 2007;11(1):1-16. doi: 10.1016/j. cld.2007.02.009. [PubMed: 17544968]

2. Matteoni CA, Younossi ZM, Gramlich T, Boparai N, Liu YC, McCullough AJ. Nonalcoholic fatty liver disease: a spectrum of clinical and pathological severity. Gastroenterology.1999;116(6):1413-9. [PubMed: 10348825]

3. Ratziu V, Giral P, Charlotte F, Bruckert E, Thibault V, Theodorou I, et al. Liver fibrosis in overweight patients. Gastroenterology. 2000;118(6):1117-23. [PubMed:10833486]

4. Musso G, Gambino R, Cassader M, Pagano G. Meta-analysis: natural history of non-alcoholic fatty liver disease (NAFLD) and diagnostic accuracy of non-invasive tests for liver disease severity. Ann Med. 2011;43(8):617-49. doi: 10.3109/07853890.2010.518623. [PubMed: 21039302]

5. Hamaguchi M, Kojima T, Takeda N, Nagata C, Takeda J, Sarui H, et al. Nonalcoholic fatty liver disease is a novel predictor of cardiovascular disease. World J Gastroenterol. 2007;13(10):1579-84. [PubMed: 17461452 ]

6. Thoma C, Day CP, Trenell MI. Lifestyle interventions for the treatment of non-alcoholic fatty liver disease in adults: a systematic review. JHepatol. 2012;56(1):255-66. doi:10.1016/j.jhep.2011.06.010. [PubMed: 21723839]

7. Eslamparast T, Eghtesad S, Poustchi H, Hekmatdoost A. Recent advances in dietary supplementation, in treating non-alcoholic fatty liver disease. World J Hepatol. 2015;7(2):204-12. doi: 10.4254/ wjh.v7.i2.204. [PubMed: 25729475]

8. Sahebkar A. Potential efficacy of ginger as a natural supplement for nonalcoholic fatty liver disease. World J Gastroenterol. 2011;17(2):271-2. doi:10.3748/wjg.v17.i2.271. [PubMed: 21246004]

9. Langner E, Greifenberg S, Gruenwald J. Ginger: history and use. Adv Ther. 1998;15(1):25-44. [PubMed:10178636]
10. Thomson M, Al-Qattan KK, Al-Sawan SM, Alnaqeeb MA, Khan I, Ali M. The use of ginger (Zingiber officinale Rosc.) as a potential anti-inflammatory and antithrombotic agent. Prostaglandins Leukot Essent Fatty Acids. 2002;67(6):475-8. [PubMed:12468270]

11. Bhandari U, Kanojia R, Pillai KK. Effect of ethanolic extract of Zingiber officinale on dyslipidaemia in diabetic rats.J Ethnopharmacol. 2005;97(2):227-30. doi: 10.1016/j.jep.2004.11.011. [PubMed: 15707757]

12. Grzanna R, Lindmark L, Frondoza CG. Ginger--an herbal medicinal product with broad anti-inflammatory actions. J Med Food. 2005;8(2):125-32. doi:10.1089/jmf.2005.8.125. [PubMed: 16117603]

13. Arablou T, Aryaeian N, Valizadeh M, Sharifi F, Hosseini A, Djalali $M$. The effect of ginger consumption on glycemic status, lipid profile and some inflammatory markers in patients with type 2 diabetes mellitus. Int J Food Sci Nutr. 2014;65(4):515-20. doi: 10.3109/09637486.2014.880671. [PubMed:24490949]

14. Shidfar F, Rajab A, Rahideh T, Khandouzi N, Hosseini S, Shidfar $S$. The effect of ginger (Zingiber officinale) on glycemic markers in patients with type 2 diabetes. $J$ Complement Integr Med. 2015;12(2):165-70. doi:10.1515/jcim-2014-0021. [PubMed:25719344]

15. Eslamparast T, Poustchi H, Zamani F, Sharafkhah M, Malekzadeh R, Hekmatdoost A. Synbiotic supplementation in nonalcoholic fatty liver disease: a randomized, double-blind, placebo-controlled pilot study. Am J Clin Nutr. 2014;99(3):535-42. doi:10.3945/ ajcn.113.068890. [PubMed: 24401715]

16. Matthews DR, Hosker JP, Rudenski AS, Naylor BA, Treacher DF, Turner RC. Homeostasis model assessment: insulin resistance and beta-cell function from fasting plasma glucose and insulin concentrations in man. Diabetologia. 1985;28(7):412-9. [PubMed: 3899825]

17. Mozaffari-Khosravi H, Talaei B, Jalali BA, Najarzadeh A, Mozayan MR. The effect of ginger powder supplementation on insulin resistance and glycemic indices in patients with type 2 diabetes: a randomized, double-blind, placebo-controlled trial. Complement Ther Med. 2014;22(1):9-16. doi:10.1016/j.ctim.2013.12.017. [PubMed: 24559810]

18. Valenti L, Fracanzani AL, Dongiovanni P, Santorelli G, Branchi A, Taioli E, et al. Tumor necrosis factor alpha promoter polymorphisms and insulin resistance in nonalcoholic fatty liver disease. Gastroenterology. 2002;122(2):274-80. [PubMed: 11832442]

19. Larter CZ, Farrell GC. Insulin resistance, adiponectin, cytokines in NASH: Which is the best target to treat? J Hepatol. 2006;44(2):25361. doi:10.1016/j.jhep.2005.11.030. [PubMed: 16364488]

20. Farrell GC, Larter CZ. Nonalcoholic fatty liver disease: from steatosis to cirrhosis. Hepatology. 2006;43(2 Suppl 1):S99-s112. doi: 10.1002/hep.20973. [PubMed:16447287]

21. Lee TY, Lee KC, Chen SY, Chang HH. 6-Gingerol inhibits ROS and iNOS through the suppression of PKC-alpha and NF-kappaB pathways in lipopolysaccharide-stimulated mouse macrophages. Biochem Biophys Res Commun. 2009;382(1):134-9. doi: 10.1016/j. bbrc.2009.02.160. [PubMed:19268427]

22. Habib SH, Makpol S, Abdul Hamid NA, Das S, Ngah WZ, Yusof YA. Ginger extract (Zingiber officinale) has anti-cancer and anti-inflammatory effects on ethionine-induced hepatoma rats. Clinics (Sao Paulo). 2008;63(6):807-13. [PubMed:19061005]

23. Yang SQ, Lin HZ, Lane MD, Clemens M, Diehl AM. Obesity increases sensitivity to endotoxin liver injury: implications for the pathogenesis of steatohepatitis. Proc Natl Acad Sci U S A. 1997;94(6):2557-62. [PubMed: 9122234]

24. Motawi TK, Hamed MA, Shabana MH, Hashem RM, Aboul Naser AF. Zingiber officinale acts as a nutraceutical agent against liver fibrosis. Nutr Metab (Lond). 2011;8:40. doi: 10.1186/1743-7075-8-40. [PubMed: 21689445]

25. Liu CT, Raghu R, Lin SH, Wang SY, Kuo CH, Tseng YJ, et al. Metabolomics of ginger essential oil against alcoholic fatty liver in mice. J Agric Food Chem. 2013;61(46):11231-40. doi: 10.1021/jf403523g. [PubMed: 24171385]

26. Abdulaziz Bardi D, Halabi MF, Abdullah NA, Rouhollahi E, Hajrezaie M, Abdulla MA. In vivo evaluation of ethanolic extract of Zingiber officinale rhizomes for its protective effect against liver cirrhosis. Biomed Res Int. 2013;2013:918460. doi: 10.1155/2013/918460. [PubMed: 24396831] 